\title{
Consciencia y constitucionalización de los derechos humanos en Latinoamérica
}

Por Danny Ramírez Ayérdiz ${ }^{1}$

Recibido: 05.08.2015/ Aprobado: 11.08.2015

\section{RESUMEN}

La consciencia por los derechos humanos en nuestra región, solo surgió de sociedades desesperadas por la democracia y así, los derechos humanos, emergieron como fundamento de los pactos sociales de estas democracias que llevaron a la refundación política de los estados latinoamericanos. La democracia y su nuevo eje paradigmático, los derechos humanos, tomó el lugar de las dictaduras a partir de nuestros propias traumas: los más de cincuenta mil muertos y desaparecidos en el genocidio indígena de la Guatemala de Ríos Montt, los treinta mil desaparecidos en Argentina de Videla, los miles de represaliados en el Chile de Pinochet, de Banzer en Bolivia, de Stroessner en Paraguay o en la Nicaragua, ahora libre, de la dinastía de los Somoza.

Palabras clave: Constitución política, derechos humanos, movimiento de consciencia, democracia, dictaduras.

\begin{abstract}
Human rights awareness in our region, only emerged from desperate societies for democracy and thus, human rights emerged as the basis of social pacts of these democracies that led to the re-founding of Latin American States. Democracy and its new paradigmatic axis, human rights, took the place of the dictatorships from our own traumas: more than fifty thousand dead and missing in the indigenous genocide of the Cuatemala's Rios Montt, the thirty thousand disappeared in Argentina's Videla, the thousands of victims of reprisal in the Chile of Pinochet, of Banzer in Bolivia, Stroessner in Paraguay or in the Nicaragua, now free of the Somoza dynasty.
\end{abstract}

Key words: Constitution, human rights, awareness movement, democracy and dictatorships.

\section{¿A qué nos referimos con derechos humanos?}

$\mathrm{C}$

uando hablamos de derechos humanos, ciertas veces se piensa en que son cuestiones abstractas, cosas que dicen de repente los juristas y filósofos, discusiones morales o, para quienes han perdido la fe en la justicia y en la lucha por la igualdad, simples palabras impresas, ideas locas puestas en las leyes, en la Constitución o en los tratados internacionales.

Los derechos humanos, muchos insertos en la constitución política, son en verdad todas las necesidades jurídicas, espirituales y materiales con las que cada quien debería contar para su existencia plena y digna; de tal manera, que si alguna vez en su casa faltó el pan, si una vez fue agredido, detenido injustamente por la policía, si fue sujeto de un acto de corrupción o las autoridades judiciales le negaron justicia, ahí hubo una violación de derechos humanos.

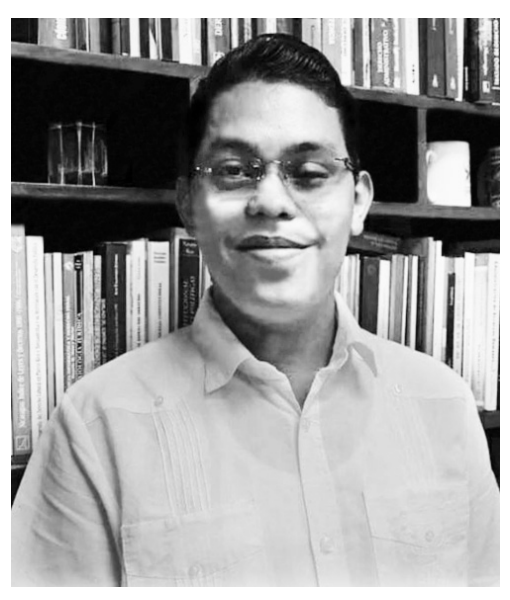

1 Docente investigador del Instituto Centroamericano de Estudios Jurídicos y Políticos, ICEJP-UPOLI. Profesor adjunto de derechos humanos y derecho internacional público en la Escuela de Ciencias Jurídicas y Políticas de la Universidad Politécnica de Nicaragua, UPOLI. Magíster en derechos humanos y democratización en América Latina y El Caribe por la Universidad Nacional de San Martín, Argentina. Correos de contacto: dannyramirezayerdiz@gmail.com - investigador-icejp2@upoli.edu.ni 
Los derechos humanos son, ciertamente, los requisitos necesarios para la realización de cada individuo según sus legítimos fines y las condiciones para el avance justo de las colectividades, en especial, las carenciadas en cualquier sentido. ${ }^{2}$

Como podemos ver, los derechos humanos atraviesan y permean la autorrealización individual y el bienestar colectivo. $Y$ es que estamos en el contexto de la sociedad de los derechos, sí, una sociedad donde cada vez los derechos humanos tienen que ver con todo. Son un fenómeno abarcador, se van metiendo en todos lados y donde entran se convierten en nuevas lógicas de lucha, de esa lucha en cuyo centro está siempre la persona humana y los grupos sociales históricamente discriminados y oprimidos. No obstante, ese fenómeno abarcador, esta sociedad de derechos, no apareció de la nada.

De la Carta Magna $^{3}$ firmada por presión por el rey Juan sin Tierra en 1215, a la Declaración de Derechos del Hombre y del Ciudadano en $1789^{4}$ o hasta la Declaración Universal de Derechos Humanos ${ }^{5}$ en 1948, hubo tanto tiempo que separa un hito del otro, como consciencia para poder producir estos documentos. No se produjeron repentinamente. Sus apretadas letras, se escribieron en memoria de la lucha colectiva, individual y a veces anónima de quienes gestaron los movimientos de consciencia a favor de los derechos reconocidos en estas declaraciones.

\section{Movimientos de consciencia por los derechos humanos: más allá de la solidaridad}

Quisiera que reflexionemos sobre la frase movimientos de consciencia. Si bien, somos parte de la cultura occidental y los grandes eventos históricos que ocurrieron en Europa de continuo son referencia "clásica" para contar la evolución del derecho constitucional o los derechos humanos, Latinoamérica vivió su propia historia de los derechos humanos.

Es cierto que los horrores de la Segunda Guerra Mundial, el Holocausto gestado vilmente por los nazis o las purgas soviéticas, escandalizaron al mundo entero y producto de este fatídico periodo, el orden internacional se refunda y los derechos humanos, con la firma de la Declaración Universal de Derechos Humanos en 1948, son consagrados como supuestas nuevas lógicas y motores de la comunidad internacional y que la Declaración Universal fue una expresión unánime de no repetición de los horrores. Pero no todos los Estados o las sociedades políticas actuaron en los mismos ánimos. Solidaridad y consciencia no es lo mismo. La Europa de luego de la Segunda Guerra mundial, entró en una profunda etapa de reflexión y esto lo reflejó el sistema jurídico comunitario recién nacido, al crear en 1954 el primer tribunal internacional de derechos humanos para juzgar a los Estados que violentan los derechos de sus ciudadanos. No querían, definitivamente, volver al horror. ${ }^{6}$

2 Instituto Peruano para Educación en Derechos Humanos y la Democracia, Los Derechos Humanos, $1^{\circ}$ (Lima: IPEDH, 2008), 5. Caso Velásquez Rodríguez, Sentencia de fondo, Serie B. N 2. (Corte Interamericana de Derechos Huma-nos, San José: 29 de julio de 1988).

3 La Carta Magna, fue producida por una imposición de los barones ingleses al rey Juan sin tierra. La Carta -paradigma importante de la historia constitucional- estableció interesantes límites al poder real y reconoció importantes derechos al pueblo y al individuo, estableciendo el hábeas corpus, el respeto de la propiedad, la regulación de la propiedad sucesoria, el derecho al debido proceso, algunas garantías procesales entre otros.

4 La teoría de la llustración de considerar la superioridad de los derechos naturales, por encima de todo acto de los gobernantes y de que éstos son consustanciales al ser humano, quedó petrificada en uno de los actos legislativos más referenciados de la evolución de los derechos humanos y de la Historia Universal misma: La Declaración de Derechos del Hombre y el Ciudadan2o, adoptada por la Asamblea Nacional francesa el 26 de agosto de 1789; no obstante, su ratificación por el rey Luis XVI se hizo efectiva hasta el 5 de octubre del mismo año por las presiones de los diputados de la Asamblea y del pueblo que se desplazó a Versalles a exigir su sanción. Con la Declaración francesa, empieza la etapa positivizadora de los derechos humanos (Erwin Silva, Derechos Humanos. Historia, fundamentos y textos, $2^{\circ}$ ed (Managua: Nos-Otros, 2004), 5).

5 La Declaración Universal, aprobada por la Asamblea General de las Naciones Unidas en 10 de diciembre de 1948, es un documento, cuya fuerza y trascendencia internacional es tan sentida, que en sí, no es instrumento vinculante, es decir, que obligue a los Estados a cumplirlo; no obstante, el alcance, importancia histórica y política de sus disposiciones, han hecho que la Declaración Universal a ser considerada una norma de ius cogens, lo que le confiere la suficiente autoridad moral, tal como si surtiera los efectos de un tratado internacional.

6 La Corte Europea de Derechos Humanos se creó por voluntad del Convenio para la Protección de los Derechos Humanos y las Libertades Fundamentales, aprobado en Roma el 4 de noviembre de 1950 en el marco del Consejo de Europa. Es el principal instrumento de derechos civiles y políticos del continente europeo; además fue el primer instrumento internacional con fuerza obligatoria en materia de derechos humanos civiles y políticos, incluso anterior al Pacto Internacional de Derechos Civiles y Políticos de 1966. Su temprana firma y entrada en vigor (1958). permitió la creación y desarrollo del primer sistema regional de protección de derechos humanos, integrado por la Corte Europea de Derechos Humanos y la extinta Comisión Europea de Derechos Humanos, que cesó en sus funciones en 1998. Actualmente, se reconoce al Sistema Europeo de Derechos Humanos, creado por el Convenio de 1950, como el más eficiente y completo en comparación con sus pares, a saber, el Sistema Interamericano y el Sistema Africano de Derechos Humanos, a quienes sirvió de modelo. 
Latinoamérica, incluida Nicaragua, se solidarizó y se escandalizó por los horrores de la Segunda Guerra Mundial, pero lo dije ya: solidaridad, escándalo y consciencia son tres actitudes que generan distintas conductas unas con mayores o menores compromisos. El foco del conflicto, los millones de muertos no los puso precisamente América Latina. Aunque nuestra región dio el ejemplo al emitir la primera declaración internacional de derechos humanos en el mismo año que la Universal, incluso meses antes, aún sus sistemas políticos, sus dirigentes autoritarios no entendían lo que significaban los derechos humanos (la Declaración de Derechos del Hombre y el Ciudadano) que Europa principalmente proclamaba en 1948.

\section{Una región solidaria, convulsa}

Ese era el gran dilema de los años 50 y subsiguientes: cómo una región latinoamericana que había proclamado y apoyado la Declaración Universal de Derechos Humanos y otras iniciativas, sus sistemas políticos eran absolutamente crueles y evidentemente desprovistos de una lógica de derechos humanos. A Latinoamérica le costó un elevado, desangrado y estremecedor precio pasar de la solidaridad por los derechos humanos a gritar, en medio de la represión de las dictaduras, que necesitaba de los derechos humanos. Fue dentro de la propia sociedad, de los movimientos sociales, de los grupos que impulsaban la revolución social como salida al subdesarrollo y al entreguismo generalizado donde empezó el movimiento de consciencia por los derechos humanos, como expresión por un nuevo sentido para erradicar la injusticia imperante y sistemática.

Latinoamérica vivió su propio Holocausto, su propio Hiroshima, su propia Nagasaki en los años 60, $70 \mathrm{y}$ 80 , como consecuencia de los planes de exterminio de las dictaduras y fue en esos años donde la sociedad latinoamericana y los grupos políticos comprendieron que se necesitaba una nueva base para las tristemente pseudo democracias latinoamericanas: hablo de los derechos humanos.

La consciencia por los derechos humanos en nuestra región, sólo surgió de sociedades desesperadas por la democracia y así, los derechos humanos, emergieron como fundamento de los pactos sociales de estas democracias que llevaron a la refundación política de los estados latinoamericanos. La democracia y su nuevo eje paradigmático, los derechos humanos, tomó el lugar de las dictaduras a partir de nuestros propias traumas: los más de cincuenta mil muertos y desaparecidos en el genocidio indígena de la Guatemala de Ríos Montt, los treinta mil desaparecidos en Argentina de Videla, los miles de represaliados en el Chile de Pinochet, de Banzer en Bolivia, de Stroessner en Paraguay o en la Nicaragua, ahora libre, de la dinastía de los Somoza.

Entonces, a través de sus propias heridas, Latinoamérica comprendió que la vía para no repetir los horrores de las dictaduras -tal como los países involucrados directamente en la Segunda Guerra Mundial- era -y es aún- la democracia, la misma que botó a las dictaduras y otros fenómenos pseudemocráticos. Entonces, la sangre de los que murieron por la liberación en las dictaduras turbó a las sociedades. Y países que en realidad jamás habían experimentado una transición democrática, vivieron una genuina refundación de sus sistemas políticos. Latinoamérica y sus Estados recién autoritarios, genocidas y asesinos renovaron sus pactos sociales y la democracia después de las dictaduras, supuso una nueva lógica en la que en adelante se basaría la política: los derechos humanos como fundamento de los nuevos sistemas políticos. Antes, las propias dictaduras decían que sus sistemas despiadados eran democracias. Pero la democracia, ajena a las dictaduras militares, se aseguró que existieran verdaderas garantías para no volver a esos desastrosos gobiernos: otra vez los derechos humanos.

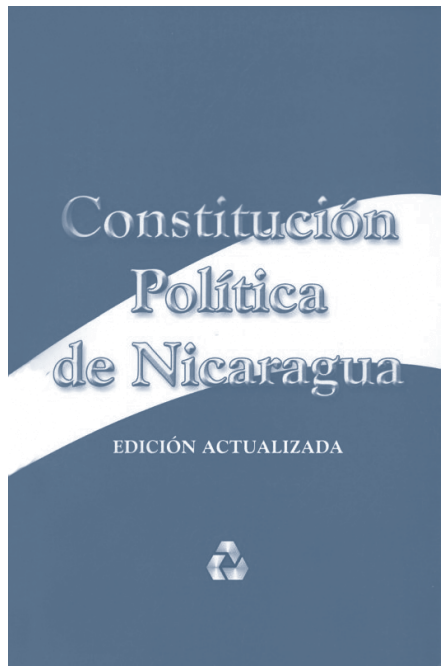

\section{La constitucionalización de los derechos humanos como garantía de no repetición}

Esta refundación de los Estados latinoamericanos de la que les hablo, producida por un hondo sentido de consciencia colectiva por vivir en democracia mediante los derechos humanos como bandera y herramientas del nuevo aparataje político, tuvo su mejor aliado en las constituciones y las reformas 


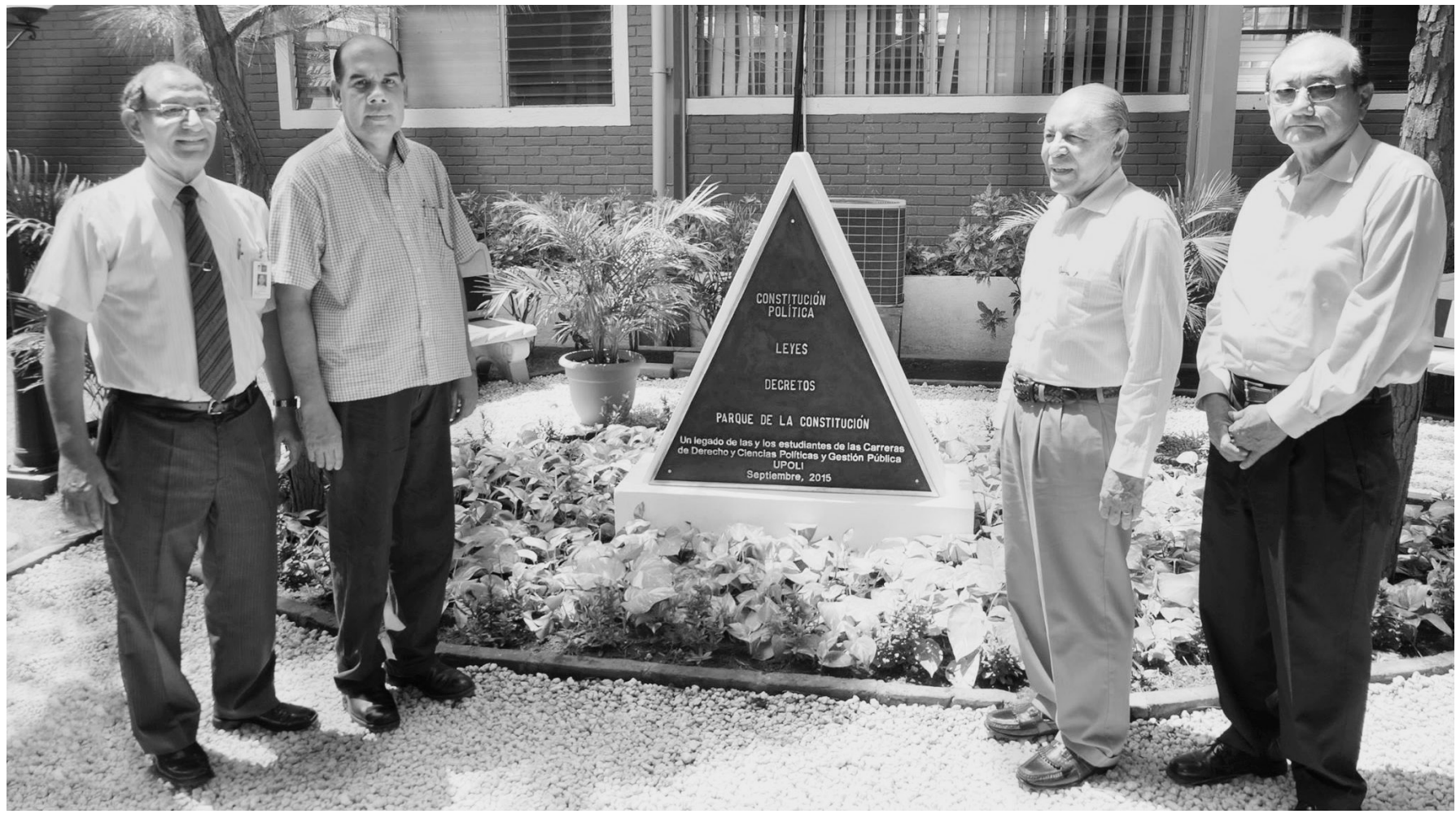

Parque de la Constitución en la UPOLI. De izquierda a derecha: MSc. Tomás Téllez, Vicerrector General; Dr. Oscar Castillo, Decano de la ECJP; Dr. Norberto Herrera, Rector Fundador de la UPOLI y Dr. Fernando Centeno, Docente de la ECJP.

constitucionales que se dictaron a lo largo de los 80 y aún no termina. La constitución, dicen los constitucionalistas, que representan la máxima voluntad del pueblo y de los acuerdos políticos hegemónicos. Entonces, suponiendo que esto es cierto, todas las reformas y constituciones dictadas luego de las dictaduras, elevan a los derechos humanos a una categoría especial que no existía en tal amplitud antes.

Las constituciones democráticas, rompen la vieja tradición de enumerar los derechos fundamentales desde la perspectiva liberal, clásica, burguesa, propietarista y les da una visión de amplitud, de universalidad, de erga omnes. Se transita del derecho fundamental clásico al derecho humano per se. Y esto no lo es todo: las constituciones democráticas post dictaduras, incluyen nuevos grupos de derechos que terminan por quebrar la perspectiva liberal de derechos fundamentales y un ejemplo a mano es la constitución revolucionaria de Nicaragua de 1987, producto de la lucha de liberación y la autodeterminación popular contra el somocismo criminal. La constitución de la Revolución va más allá de los típicos derechos individuales y sociales y consagra derechos amplios para las comunidades étnicas del caribe sur, declara la multietnicidad del Estado y le da rango constitucional a una serie de tratados de derechos humanos ratificados en su mayoría por el gobierno revolucionario. ¡Qué extraordinario! Impensable en las constituciones somocistas, que para mencionar, ni siquiera se usó la frase derechos humanos en la Constitución kupia-kumi de 1974, la última de la dinastía.

Toda una nueva lógica que desafió la manera tradicional de contrapesar el poder político. Cuando el constituyente revolucionario consagró a los derechos humanos y sus tratados internacionales que los recogen, lo hizo porque sabe que el poder corrompe y los derechos limitan esa corrupción cuando estos ya se consolidaron en sociedades ya golpeadas por dictaduras atroces a raíz de ese movimiento de consciencia que mencioné al inicio; movimientos que en Nicaragua fue heroico, contestatario y efectivo. Al consagrar los derechos humanos en la Constitución, el poder establece a la persona humana sólo por encima de la ley y sus operadores, cuando esta última es injusta, arbitraria y abusiva. Y los tratados de derechos humanos se establecen en la Constitución para que la gente sepa que a nivel internacional hay vías 
qué agotar para buscar justicia cuando los remedios y mecanismos constitucionales o legales fallan para proteger al individuo o a los grupos menos favorecidos de la sociedad.

\section{Honrar el legado de los mártires y héroes de la Revolución}

Ahora, ese casamiento entre derechos humanos y constitución política después de las dictaduras ha hecho que estos mismos avancen y lo vayan abarcando casi todo. De ahí que hoy más que nunca se necesita que se defiendan los derechos humanos que están consagrados en la constitución, porque este nuevo paradigma del que les hablé donde la democracia tiene como garantía a los derechos humanos como límites de los excesos del poder, está en peligro ante nuevos fenómenos que pretenden doblegar y quebrantar este indisoluble matrimonio entre derechos humanos y constitución. $Y$ a pesar de que existe una permanente pugna doctrinal entre internacionalistas y constitucionalistas sobre la eterna discusión si los tratados de derechos humanos son superiores o inferiores que la Constitución, en algo si existe un acuerdo firme entre unos y otros: los derechos humanos tienen que defenderse para que hablemos democracia, de estado social, democrático y constitucional de derechos o de cualquier otro sistema donde se pretenda reivindicar a la persona y a los grupos históricamente discriminados por encima de cualquier proyecto político que nos pretenda regresa a las etapas del dolor latinoamericano.

--Por tanto, si el movimiento de consciencia por los derechos humanos fue gestado desde los movimientos de revolución social y miles murieron para logarlo, ciertamente existe un deber recurrente de honrar a estos actores y actoras muchos de ellos muertos en el anonimato y que ayudaron a sentar las bases de un sistema político basado en los derechos humanos para que hoy no vivamos los exabruptos de los Somoza o en cualquier otra dictadura en Nuestramérica Latina. Es menester que nos convirtamos en operadores en defensa de nuestra Constitución y que, en lugar de perder la fe en ella por causa de los operadores políticos que habitan en las tranquilas calles del poder, recordemos que ella, para entrar en vigor en 1987, miles dieron sus vidas, desaparecieron y fueron torturados. Tal vez sin pensarlo, defendieron los derechos humanos que hoy están consagrados en la Carta Magna nuestra y las de todos los Estados de Latinoamérica.

\section{Bibliografía}

Silva, E. Derechos Humanos. Historia, Fundamentos y Textos, $2^{\circ}$ ed. Nos-Otros, Managua, 2004.

Instituto Peruano para Educación, Derechos Humanos y la Democracia en Los Derechos Humanos, $1^{\circ}$. Lima: IPEDH, 2008.

Corte Interamericana de Derechos Humanos. Caso Velásquez Rodríguez, Sentencia de fondo, Serie B. № 2., San José: 29 de julio de 1988.

Asamblea Nacional. Constitución Política de la República con reformas incorporadas publicada en la Gaceta, Diario Oficial del 18 de febrero de 2014.
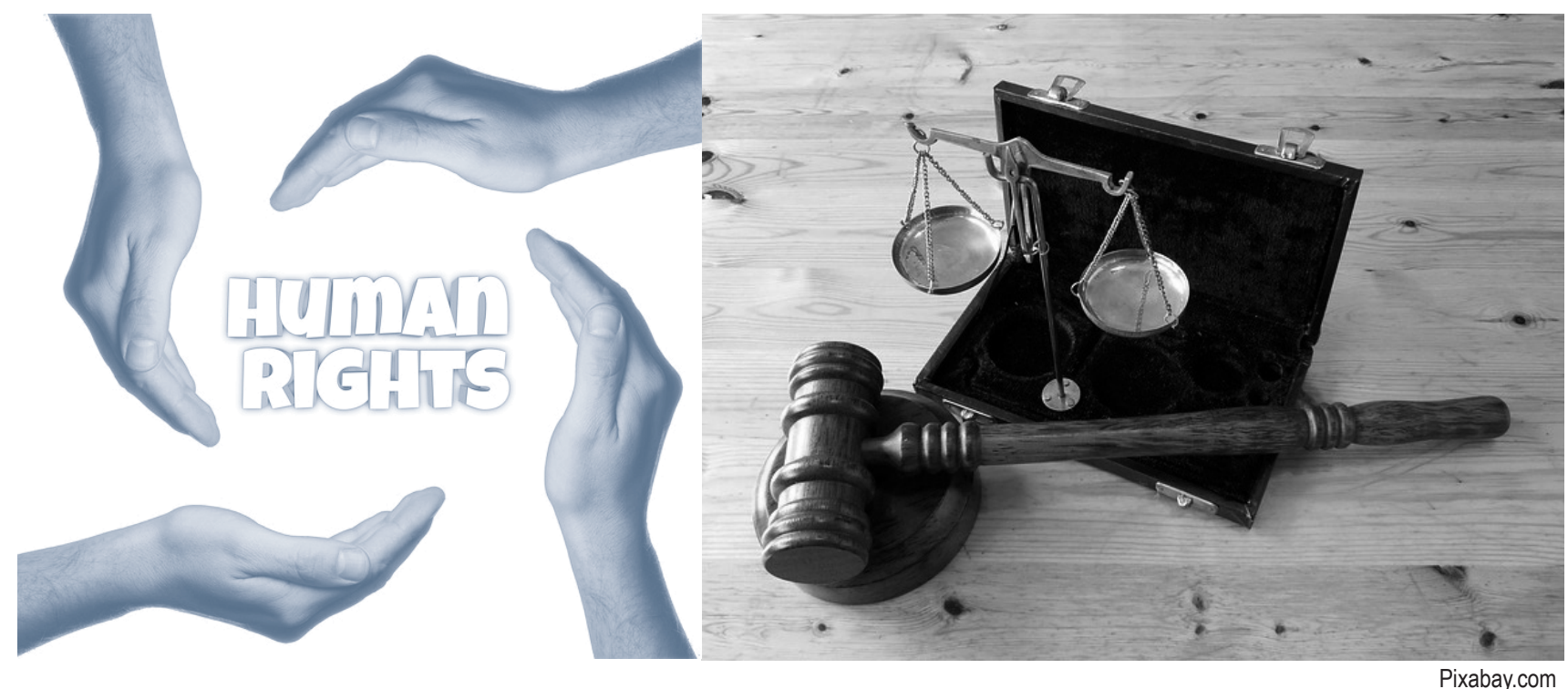